\title{
Evaluating the probiotic and therapeutic potentials of Saccharomyces cerevisiae strain (OBS2) isolated from fermented nectar of toddy palm
}

\author{
Banoth Srinivas ${ }^{1}$, Ganapathiwar Swarupa Rani ${ }^{1}$, Bhukya Kiran Kumar ${ }^{1}$, Banoth Chandrasekhar ${ }^{1}$, \\ Kommalapati Vamsi Krishna ${ }^{2}$, Tangutur Anjana Devi² and Bhukya Bhima ${ }^{1 *}$ (])
}

\begin{abstract}
The purpose of this study is to evaluate the probiotic characteristics of 15 yeast strains isolated from nectar of toddy palm. Initially, the collected samples were inoculated on yeast extract peptone dextrose agar plates and the colonies so obtained were culturally and morphologically characterized. Commercial probiotic yeast, Saccharomyces boulardii served as the control in these experiments. Of the 15 yeast strains, the isolates that were resistant to antibiotics and worked synergistically with other cultures were considered for further evaluation. Selected isolates were evaluated in vitro for tolerance to simulated gastrointestinal conditions such as temperature, $\mathrm{pH}$, bile and gastric juice. Further the yeast isolates were evaluated for their pathogenicity and adherence to intestinal epithelial cells. The 2 yeast isolates with efficient probiotic properties were finally characterized by sequencing their $5.8 \mathrm{~S} \mathrm{rRNA}$ and partial sequences of internal transcribed spacer 1 and 2. The sequences were BLAST searched in the National Center for Biotechnology Information, nucleic acid database for sequence similarity of organisms and phylogenetic evolutionary analysis was carried out. Based on maximum similarity of basic local alignment search tool results, organisms were characterized as Pichia kudriavzevii OBS1 (100\%) and Saccharomyces cerevisiae OBS2 (96\%) and sequences were finally deposited in the GenBank data library. Among these two isolates, S. cerevisiae OBS2 displayed slight/moderate antioxidant and anticancer property. Hence, strain OBS2 can be utilized and explored as a potential probiotic for therapeutic applications.
\end{abstract}

Keywords: Probiotics, Toddy nectar, Antimicrobial resistance, Therapeutics, Yeasts

\section{Introduction}

It is well known that many types of bacteria and yeast strains are being used to enhance the fermentative reactions in food industries including bread, beer, wine and xylitol (Ortiz et al. 2013). Besides fermentation, these organisms are playing numerous beneficial roles in human, animal health and nutrition. A number of previous studies reported about the use of probiotics for the prevention and treatment of gastrointestinal infections

\footnotetext{
*Correspondence: bhima.ou@gmail.com

1 Department of Microbiology, University College of Science, Osmania University, Hyderabad, Telangana 500007, India

Full list of author information is available at the end of the article
}

including food borne pathogens associated infections (Fooks and Gibson 2002). In addition, it is reported that yeast promotes both human and animal health (Zanello et al. 2009; O'Neil et al. 2012) and enhances the bioavailability of minerals through hydrolysis of phytate, folate biofortification, detoxification of mycotoxins and xenobiotics (Ohashi and Kazunari 2009). At present, the products of wild and recombinant yeast form the backbone for many commercially important sectors, including foods, beverages, pharmaceuticals, industrial enzymes and medicine (Mc Meekin et al. 1997). Saccharomyces cerevisiae has a Qualified Presumption of Safety (QPS) status (Verna and Lucak 2010; Waters et al. 2012). Potential 
probiotic mechanism of S. cerevisiae is based on emission of inhibitory proteins against pathogens, stimulation of host immunoglobulin A and exclusion of secreted toxins in host intestine (Fooks and Gibson 2002).

Tolerance to low $\mathrm{pH}$, high temperature, high ox-bile concentration, a mixture of organic acids concentration, digestive enzymes like amylase, pepsin and trypsin concentrations and ability to produce several other useful metabolites represent the beneficial characteristics of probiotics (Fridkin and Jarvis 1996). Probiotics need to survive the inevitable biological barriers of the gut. It is evidently proven from the previous in vitro studies that eukaryotic probiotics can tolerate the well simulated environmental conditions of the digestive tract (Kumura et al. 2004). The probiotic strains of S. cerevisiae are not only able to survive in the digestive tract of the host, but also found to persist at high populations (Blehaut et al. 1989). The pharmacokinetic studies indicate that the probiotic Sachcharomyces var boulardii when ingested orally achieved a steady state in the gut within 3 days, but eliminated within 2-5 days after it is discontinued. Such elimination from the digestive tract is believed to be due to the barrier effect of the complex established by resident microbiota of the gut (Fridkin and Jarvis 1996; Yoo and Kim 2016).

Yeast with its derivatives or by-products has gained huge importance and become a model organism for many biological functions in our life. S. cerevisiae is a potential organism to be explored as novel natural anticancer, antioxidant and immunostimulating agent for using in functional foods or medicine (Hassan 2011; Balasubramanian and Ragunathan 2012).

Since the droppings and exudates of toddy palm during hot summer stimulate the growth of simple sugar fermenting, high temperature $\left(\sim 40^{\circ} \mathrm{C}\right)$ resistant microorganisms (Abdel Fattah et al. 2000), in the present study, yeasts were isolated from fermented nectar of toddy palm. The isolated yeast samples were subjected to a series of analyses for efficient probiotic and therapeutic characteristics.

\section{Materials and methods}

\section{Isolation of yeast strains}

Samples were collected from naturally fermented nectar of toddy palm during mid day of hot summer $\left(\sim 40{ }^{\circ} \mathrm{C}\right)$ from different regions of Telangana State, India. Commercial probiotic yeast $S$. boulardii (Econorm, Dr. Reddy's Laboratories, Hyderabad, India) was used as a referral organism.

Isolation of yeasts was carried out by using standard methodology described by Tikka et al. (2013) and Zaky et al. (2014). After incubation, colonies were selected based on morphology and sub-cultured on yeast extract peptone dextrose (YPD) agar slants for further observations.

\section{Growth and characterization of yeast isolates}

Isolates were characterized by their cultural and morphological characteristics following the protocols as described by Reis et al. (2013). After incubation, cultural and morphological characteristics were observed by visible growth pattern followed by simple staining and microscopic observation.

The sugar utilization ability of yeast isolates was achieved by their sugar assimilation profiling. The sugar assimilation property enables us to determine the ability of yeast to use a specific sugar/carbohydrate as a sole source of carbon. Yeast nitrogen base (YNB) medium with $2 \%(\mathrm{w} / \mathrm{v})$ specific selected sugar (s) like glucose, maltose, ribose, fructose, xylose, cellobiose, dextrose, mannose, starch, sucrose, lactose and galactose were used for determination of yeast sugar assimilation property on the basis of biomass formation (Marinho et al. 2010).

\section{Assessment for probiotic properties Antimicrobial drug sensitivity}

The yeast isolates were tested for antimicrobial drug sensitivity against 8 antifungal agents like Cefmetazole $30 \mu \mathrm{g}$ (CMZ 30), Ceftazidime $10 \mu \mathrm{g}$ (CTR 10), Clotrimazole $10 \mu \mathrm{g}$ (CC 10), Fluconazole $10 \mu \mathrm{g}$ (FLC 10), Itraconazole $10 \mu \mathrm{g}$ (IT 10), Ketoconazole $50 \mu \mathrm{g}$ (KT 50), Miconazole $50 \mu \mathrm{g}$ (MIC 50) and Nystatin $100 \mu \mathrm{g}$ (NS 100) and 20 antibacterial agents like Amoxicillin $10 \mu \mathrm{g}$ (AMX 10), Ampicillin $10 \mu \mathrm{g}$ (AMP 10), Ampicillin $25 \mu \mathrm{g}$ (AMP 25), Bacitracin $10 \mu \mathrm{g}$ (B 10), Ceftriaxone $10 \mu \mathrm{g}$ (CT 10), Chloramphenicol $10 \mu \mathrm{g}$ (C 10), Clindamycin $10 \mu \mathrm{g}$ (CD 10), Cloxacillin $10 \mu \mathrm{g}$ (COX 10), Enrofloxacin $10 \mu \mathrm{g}$ (EX 10), Erythromycin $10 \mu \mathrm{g}$ (E 10), Gentamicin $10 \mu \mathrm{g}$ (GEN 10), Kanamycin $30 \mu \mathrm{g}$ (K 30), Lincomycin $10 \mu \mathrm{g}$ (L 10), Methicillin $10 \mu \mathrm{g}$ (MET 10), Neomycin $10 \mu \mathrm{g}$ (N 10), Norfloxacin $10 \mu \mathrm{g}$ (NX 10), Penicillin G $10 \mu \mathrm{g}$ (P 10), Streptomycin $10 \mu \mathrm{g}$ (S 10), Streptomycin $25 \mu \mathrm{g}$ (S 25), Teracycline $10 \mu \mathrm{g}$ (TE 10), Teracycline $30 \mu \mathrm{g}$ (TE 30), Trimethoprim $10 \mu \mathrm{g}$ (TR 10) and Vancomycin $10 \mu g$ (VA 10) of Sigma Aldrich (Sigma Aldrich Pvt. Ltd., India) were placed on yeast inoculated YPD medium plates and incubated for $48 \mathrm{~h}$ at $30{ }^{\circ} \mathrm{C}$. After incubation drug sensitivity of the yeast isolates was evaluated by measuring the zone of inhibition (Mashad et al. 2012).

\section{Antagonistic activity against pathogens}

Antagonistic activity of yeast isolates was evaluated by agar well plate method by measuring the zone of inhibition around the wells as per protocol described by Sibanda et al. (2010) against human intestinal pathogenic 
bacteria like Escherichia coli 0157:H7, Pseudomonas aeruginosa, Klebsiella pneumonia, Staphylococcus aureus, Salmonella typhi and Salmonella paratyphi.

\section{Co-culture activity with healthy commensals}

Co-culture activity assay is yet another method to determine the positive or negative impact and/or ability of candidate probiotic yeast isolates to coexist with normal bacterial flora of the human intestine. Yeast isolates and bacterial cultures like E. coli 0150:H5, Lactobacillus acidophilus were activated in their basal media and equal volumes $(0.1 \mathrm{ml})$ of their suspensions were inoculated in a modified medium ( $1 \%$ yeast extract, $1 \%$ peptone, $0.25 \%$ $\mathrm{NaCl}$ and $1 \%$ dextrose) with $\mathrm{pH} 6.5$ and incubated at $37^{\circ} \mathrm{C}$ for $24 \mathrm{~h}$. After incubation, culture suspensions were diluted to tenfold and $10 \mu \mathrm{l}$ of $10^{-3}$ dilution was inoculated on YPD agar medium with Ampicillin $(30 \mu \mathrm{g} / \mathrm{ml})$, Kanamycin $(30 \mu \mathrm{g} / \mathrm{ml})$ for yeast growth and nutrient agar medium with Geneticin $(200 \mu \mathrm{g} / \mathrm{ml})$ for bacterial growth. The YPD agar medium plates were incubated at $30^{\circ} \mathrm{C}$ for $48 \mathrm{~h}$ and nutrient agar plates were incubated at $37^{\circ} \mathrm{C}$ for $24 \mathrm{~h}$. After incubation, percent viability of yeast isolates and bacteria was determined on the basis of colony forming units (CFU) using modified methodology of Paschos et al. (2015).

\section{Stress tolerance}

A crucial step towards the identification and selection of potential probiotic candidates is to evaluate their resistance to the extreme conditions of the gastrointestinal tract. The barriers that must be overcome are temperature, low $\mathrm{pH}$, organic acids and digestive enzymes, i.e. pepsin and amylase in the stomach and trypsin and bile in the upper intestine (Corzo and Gilliland 1999). The probiotic organism should be able to tolerate high temperature, i.e. above $37{ }^{\circ} \mathrm{C}$ and the diverse conditions of the gastric juice. Therefore, to test the parameters of stress tolerance of yeast isolates the following experiments were performed.

\section{Resilience to gastrointestinal parameters}

Actively growing yeast suspensions $(1 \mathrm{ml})$ were inoculated in $100 \mathrm{ml}$ phosphate buffered saline (PBS) and incubated for $4 \mathrm{~h}$ at different temperatures (30,37, 40 and $\left.45{ }^{\circ} \mathrm{C}\right)$, with different $\mathrm{pH}(2.0,2.5,3.0$ and 3.5), different percentages of ox bile $(0.25,0.5,0.75$ and 1.0$)$ and different percent mixture of 3 organic acids like propionic, butyric and acetic acids in 7:2:1 ratio $(0.25,0.5,0.75$ and 1.0). After incubation, $100 \mu \mathrm{l}$ of inoculum from each set was inoculated on YPD agar at $30^{\circ} \mathrm{C}$ for $24 \mathrm{~h}$ and percent tolerance was calculated by using the formula $(\log N / \log$ $\left.N_{0}\right) \times 100$ where $\mathrm{N}$ is count after incubation and $\mathrm{N}_{0}$ is count before incubation (both expressed as cfu/ml) (Syal and Vohra 2013).

Aqueous solution ( $1 \mathrm{ml}$ ) of $5 \% \mathrm{NaCl}$ was taken to create an in vitro gastric environment with different concentrations of enzymes like Pepsin (2.0, 4.0, and $6.0 \mathrm{~g} \mathrm{l}^{-1}$ ), Trypsin (0.25, 0.5 and $0.75 \mathrm{mg} \mathrm{ml}^{-1}$ ) and Amylase (250, 300 and $350 \mathrm{IU} \mathrm{ml}^{-1}$ ) (Pennacchia et al. 2008). The $\mathrm{pH}$ of pepsin containing solution was adjusted to 2.0 and trypsin and amylase containing solution was adjusted to 7.5. All the sets were inoculated with $0.1 \%$ inoculum of yeast isolates and incubated at $30{ }^{\circ} \mathrm{C}$ in a shaking incubator at $150 \mathrm{rpm}$ for $4 \mathrm{~h}$. After incubation of all the sets, $100 \mu \mathrm{l}$ of inoculum from each set was inoculated on YPD agar medium for the determination of percent tolerance of yeast isolates (Syal and Vohra 2013).

\section{Pathogenicity of yeast isolates}

Yeast such as $S$. boulardii are best studied probiotics being used successfully and well known to play a crucial role in the treatment and management of diarrhoea and inflammatory bowel disease and to reduce the duration of disease. Despite an excellent record of safe use, yeasts may still be the cause of localized infections in some patients.

Therefore, in order to determine whether the yeast isolates show pathogenicity, it was studied by the detection of enzymes like protease, phospholipase, the ability of haemolysis and by detection of specific pathogenic genes through polymerase chain reaction (PCR) amplification. Protease activity was tested using milk casein and gelatin as substrates by the method described by Lechuga et al. (2016) and was adapted to verify the phospholipase activity. Similarly, haemolytic activity was determined by a modified method of Manns et al. (1994). Protease and phospholipase production was detected by halos formation around the culture and haemolysin activity was detected by measurement of haemolytic index (Luo et al. 2001). Extracted genomic DNA of each isolate was amplified with universal primer SR6R (5'-AAGTAAAGT-CGTAACAAGG-3') and LR1 (5'-GGTTGGTTTCTTTTCCT-3') of human pathogenic Candida albicans. The reaction mixture for PCR and conditions are as per protocol described by Koehn (1982). The amplicons formed as PCR product were visualized on an agarose gel.

\section{Cell adhesion}

Caco-2 cells derived from human intestinal epithelial cells were purchased from National Centre for Cell Science (NCCS), Pune, India and maintained in Eagle's minimal essential medium (MEM) with glutamine $(0.584 \mathrm{~g} / \mathrm{l})$, sodium bicarbonate $(3.7 \mathrm{~g} / \mathrm{l})$, penicillin $(100 \mathrm{U} / \mathrm{ml})$, 
streptomycin $(100 \mu \mathrm{g} / \mathrm{ml})$, and $5 \%$ serum and the final $\mathrm{pH}$ of medium was adjusted to 7.2 before sterilization. Culture flasks with medium were maintained in a $\mathrm{CO}_{2}$ incubator at $37{ }^{\circ} \mathrm{C}$ and $5 \% \mathrm{CO}_{2}$ as per the protocol described in Nikolic et al. (2012). Inoculated monolayers were washed thrice with warm MEM medium without serum. Adhesion of yeast cells with monolayer was fixed with cold methanol, stained with Giemsa stain and observed under microscope for adherence. The mean number of yeast cells was determined and the adherence score was expressed for each yeast isolate.

\section{Molecular characterization}

Based on the probiotic characteristics, 2 yeast isolates OBS1 and OBS2 were selected and characterized by sequencing of $5.8 \mathrm{~S}$ rRNA and internal transcribed spacer (ITS) 1 and 2 (Fujita et al. 2001). Genomic DNA was extracted using an Insta Gene Matrix (BIO RAD, California, USA). The universal primers used for amplification of 5.8S rRNA, ITS1and ITS2 were $5^{\prime}$-TCCGTAGGTGAACCTGCGG-3' and $5^{\prime}$-TCCTCCGCTTATTGATATGC-3'. The PCR conditions were set to 1 min each for denaturation at $95{ }^{\circ} \mathrm{C}$, annealing at $55{ }^{\circ} \mathrm{C}, 2 \mathrm{~min}$ for an extension at $72{ }^{\circ} \mathrm{C}$ and finally finishing with a 10 min step at $72{ }^{\circ} \mathrm{C}$. The amplicons were purified with a multiscreen filter plate (Millipore Corp., Bedford, MA, USA) and sequencing was performed using PRISM BigDye Terminator v3.1 Cycle sequencing Kit (Applied Biosystems, California, USA). The amplicons were added to Hi-Di formamide (Applied Biosystems, Foster City, CA) and the mixture was incubated at $95^{\circ} \mathrm{C}$ for $5 \mathrm{~min}$, followed by incubation on ice for $5 \mathrm{~min}$ and then analyzed by ABI Prism 3730XL DNA analyzer (Applied Biosystems). Complete sequence of 5.8S rRNA and partial sequences of internal transcribed spacer (ITS) 1 and 2 were BLAST searched in NCBI database (www.ncbi.nlm.nih.gov) for similarity. Based on maximum similarity of BLAST results, a phylogenetic dendrogram was constructed using Phylip 3.69.

\section{Anticancer properties}

Three test samples were prepared to assess the cytotoxic effect of yeast isolates on the chosen cancer cell lines MCF7 (Breast cancer) and IMR32 (Neuroblastoma). Among three samples, two were prepared by cultivating yeast isolates OBS1 and OBS2 separately overnight and one without yeast in YPD broth medium. After overnight incubation, all the three samples were centrifuged to separate yeast cells and supernatants were filtered using membrane filters to obtain clear sterile test samples. Sulforhodamine B (SRB) assay was performed to assess the anticancer property of yeast isolates (Szajewska et al. 2006). Briefly, $10 \times 10^{3}$ cells in $100 \mu \mathrm{l}$ Dulbecco's modified Eagle medium (DMEM) per well were seeded in triplicates in 96-well flat-bottom plates and are allowed to adhere overnight. Following this incubation period, the filtrate obtained from cultured broth of yeast isolates OBS1 and OBS2 were added to each of the wells in a volume dependent manner $(2-40 \mu \mathrm{l})$ and incubated for $48 \mathrm{~h}$. After $48 \mathrm{~h}$, the media was replaced with $100 \mu \mathrm{l}$ of $10 \%$ Trichloroacetic acid for fixation of cells for $1 \mathrm{~h}$ at $4{ }^{\circ} \mathrm{C}$ and stained with $0.4 \%$ sulforhodamine B dissolved in $1 \%$ acetic acid. Cells were then washed with $1 \%$ acetic acid to remove unbound dye. The protein-bound dye was extracted with $10 \mathrm{mM}$ Tris base to determine the optical density at $510 \mathrm{~nm}$ wavelength.

\section{Antioxidant properties}

The yeast autolysate for determination of antioxidant activity was prepared as per the protocol described by Chana et al. (2005). Cell pellets of two active yeast isolates, OBS1 and OBS2 were suspended in $20 \mathrm{ml}$ sterile distilled water and incubated at $25^{\circ} \mathrm{C}$ for $72 \mathrm{~h}$. After incubation, the content was centrifuged and the volume of separated supernatant autolysate solution was made up to $500 \mathrm{ml}$ with distilled water. The antioxidant activity of yeast isolates was assayed using phosphomolybdenum method (Kumaran and Karunakaran 2007). The increased absorbance of reaction mixture indicates the increased antioxidant property.

\section{Statistical analysis}

Statistical analysis was carried out for the data using the Statistical Package for the Social Sciences (SPSS), version 12.0 for Windows, for the determination of average and standard deviations. In this analysis, independent variables are temperature, $\mathrm{pH}$, ox-bile, organic acid mixture, gastric enzymes like amylase, trypsin and pepsin and the dependent variables are the percent tolerance of yeast isolates. Significance was determined to be $\mathrm{p}<0.01$ using two-way analysis of variance (ANOVA), followed by Duncan's multiple range test.

\section{Sequence and culture deposition}

Sequences were submitted to the NCBI GenBank and acquired accession numbers for both the strains. OBS1: KP998095 and OBS2: KP998094. Based on probiotic and therapeutic properties, yeast OBS2 was selected and deposited into DSMZ culture collection as S. cerevisiae DSM 103642. 


\section{Results}

Isolation and associated growth/morphological characteristics of yeast strains

A total of 53 yeast isolates (OBS1-OBS53) were isolated from the nectar of toddy palm collected from different regions of Telangana state of India. The basic idea behind this is to enable isolation of yeast strains from different hostile environments which will facilitate the function of isolated strains with plausible efficient probiotic characteristics.

Fifteen yeast isolates (OBS1-OBS15) were selected for further studies based on their differential growth and colony morphology like margin, elevation, color, type of fermentation and shape.

The yeast isolate OBS12 showed biomass formation upon mannitol supplementation, S. boulardii showed biomass formation in Lactose supplemented medium. However, all isolates did not form biomass in ribose, cellulose, starch supplemented media. Except OBS9, OBS2 and OBS4, biomass was observed for all the isolates in galactose supplemented medium (Table 1).

\section{Probiotic properties of yeast isolates} Antimicrobial drug sensitivity and antagonistic properties Fifteen yeast isolates were tested for antimicrobial drug susceptibility by disc diffusion method. The results of the antimicrobial drug susceptibility of the yeast strains OBS1 and OBS2 against 21 antibacterial and 8 antifungal drugs are summarized in Fig. 1. All the 15 isolates were resistant to the $\beta$ - lactams cephalosporins i.e. Cefmetazole $(30 \mu \mathrm{g})$ and Ceftazidime $(10 \mu \mathrm{g})$; OBS1 and OBS10 were resistant to the cell wall biosynthesis inhibitor and the antifungal agent Clotrimazole $(10 \mu \mathrm{g}) ; S$. boulardii, OBS4 and OBS10 were resistant to synthetic Triazole antifungal agent Fluconazole $(10 \mu \mathrm{g}) ; \mathrm{S}$. boulardii, OBS1, OBS2, OBS3, OBS5, OBS10, OBS13 and OBS15 were resistant to azole antifungal, Itraconazole $(10 \mu \mathrm{g})$ and OBS9 was resistant to Ketoconazole $(50 \mu \mathrm{g})$, Miconazole $(50 \mu \mathrm{g})$, the azole antifungals and Nystatin, an antimycotic polyene antibiotic $(100 \mu \mathrm{g})$. The other isolates were sensitive to all the antifungal agents. Yeast isolate OBS2 was sensitive to aminoglycosides-Streptomycin $(10 \mu \mathrm{g})$ and Gentamicin $(10 \mu \mathrm{g})$ with 0.1 and $0.2 \mathrm{~cm}$ zone of inhibition, respectively whereas OBS1 was sensitive to Gentamicin $(10 \mu \mathrm{g})$ with $0.2 \mathrm{~cm}$ zone of inhibition. All other isolates were resistant to all antibacterial agents and hence did not show any zone of inhibition around the disc.

Based on the results of morphological, cultural and antimicrobial drug sensitivity profiles, 8 isolates (i.e. OBS1, OBS2, OBS4, OBS7, OBS9, OBS11, OBS12 and $S$. boulardii) were selected for further studies.

The results clearly indicate that $12 \mathrm{~h}$ grown cultures of all the eight yeast isolates and $24 \mathrm{~h}$ grown cultures of OBS7 and OBS9 did not show any antimicrobial/antagonistic activity towards intestinal pathogenic bacteria. Pathogenic E. coli 0157:H7 was resistant to S. boulardii and OBS4 whereas K. pneumoniae was resistant to $24 \mathrm{~h}$ grown cultures of OBS1. Bacterial cultures like $E$. coli 0157:H7, P. aeruginosa, K. pneumonia, Staph. aureus, $S$. typhi and $S$. paratyphi were sensitive to yeast isolates OBS1 with $0.8,0.20 .0,0.8,0.3$ and $0.8 \mathrm{~cm}, \mathrm{OBS} 2$ with

Table 1 Sugar assimilation profile of yeast isolates

\begin{tabular}{|c|c|c|c|c|c|c|c|c|c|c|c|c|c|c|}
\hline S. no & Isolate & $A$ & B & $C$ & D & $E$ & $F$ & G & $\mathrm{H}$ & I & $J$ & $\mathrm{~K}$ & $\mathrm{~L}$ & $\bar{M}$ \\
\hline 1 & S. boulardii & + & + & + & - & + & - & - & - & + & + & + & + & - \\
\hline 2 & OBS1 & + & + & + & - & + & - & - & - & + & + & - & + & - \\
\hline 3 & OBS2 & + & + & + & - & + & - & - & - & + & + & - & - & - \\
\hline 4 & OBS3 & + & + & + & - & + & - & - & - & + & + & - & + & - \\
\hline 5 & OBS4 & + & + & - & - & + & - & - & - & + & + & - & - & - \\
\hline 6 & OBS5 & + & + & + & - & + & - & - & - & + & + & - & + & - \\
\hline 7 & OBS6 & + & + & + & - & + & - & - & - & + & + & - & + & - \\
\hline 8 & OBS7 & + & + & + & - & + & - & - & - & + & + & - & + & - \\
\hline 9 & OBS9 & + & + & + & - & + & - & - & - & + & + & - & - & - \\
\hline 10 & OBS 10 & + & + & + & - & + & - & - & - & + & + & - & + & - \\
\hline 11 & OBS12 & + & + & + & - & + & + & - & - & + & + & - & + & - \\
\hline 12 & OBS13 & + & + & + & - & + & - & - & - & + & + & - & + & - \\
\hline 13 & OBS14 & + & + & + & - & + & - & - & - & + & + & - & + & - \\
\hline
\end{tabular}

$A$ glucose, $B$ fructose, $C$ maltose, $D$ ribose, $E$ dextrose, $F$ mannitol, $G$ cellulose, $H$ starch, $I$ xylose, $J$ sucrose, $K$ lactose, $L$ galactose, $M$ control 


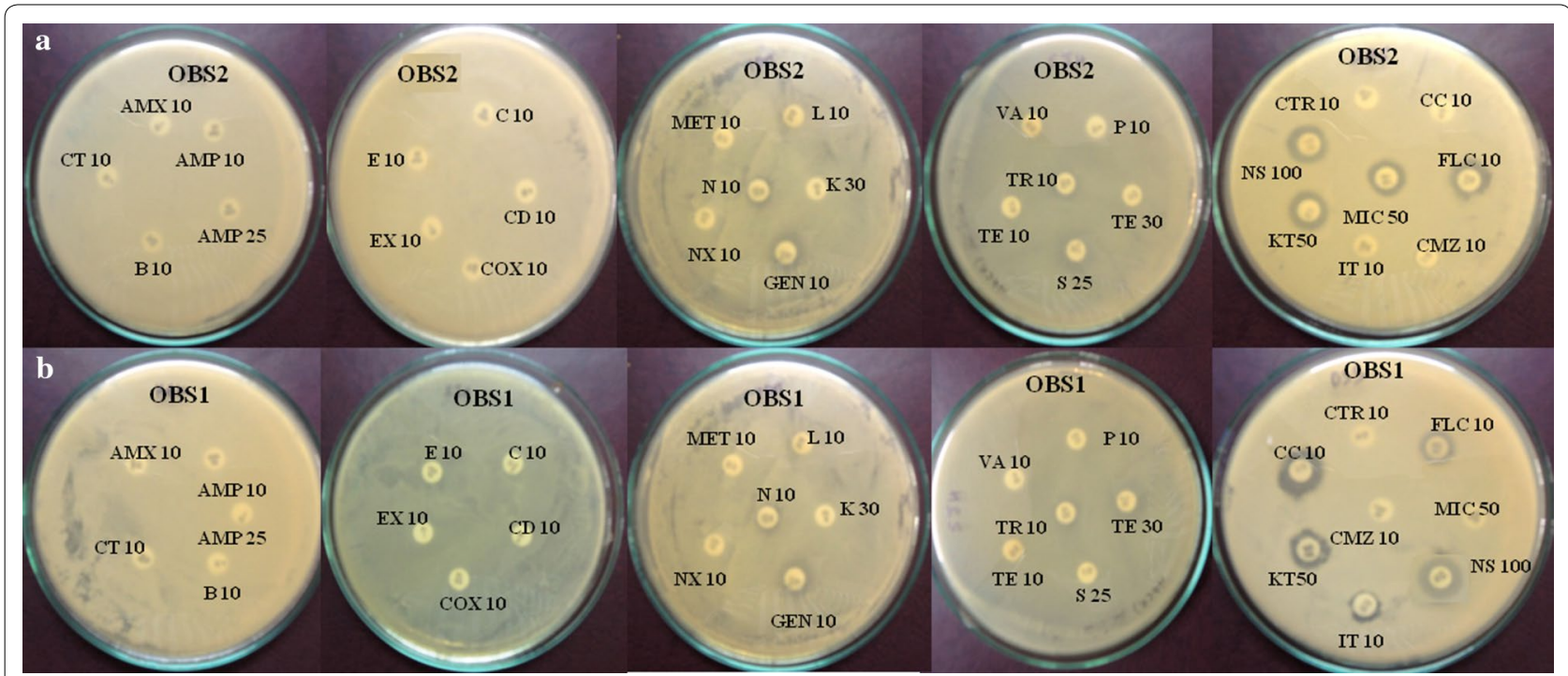

Fig. 1 Antimicrobial drug sensitivity of a yeast isolate OBS2 and $\mathbf{b} \mathrm{OBS1}$

$0.5,0.5,0.6,0.8,0.3$ and $0.8 \mathrm{~cm}, \mathrm{OBS} 3$ with $0.8,0.3,0.8$, $0.6,0.3$ and $0.8 \mathrm{~cm}$, OBS4 with $0.0,0.3,0.2,0.5,0.3$ and $0.8 \mathrm{~cm}$ and OBS12 with $1.0,0.5,1.0,1.0,0.2$ and $1.0 \mathrm{~cm}$ zone of inhibition, respectively (Fig. 2).

Yeast isolates OBS1, OBS2, OBS3, OBS4, OBS7, OBS9 and OBS12 showed percent cell viability of $78,85,81$, $78,28,35$ and 62, respectively when cultured with $E$. coli 0150:H5. Similarly the isolates OBS1, OBS2, OBS3, OBS4, OBS7, OBS9 and OBS12 showed percent cell viability of $53,82,25,28,25,26$ and 73, respectively when cultured with $L$. acidophilus. Whereas $S$. boulardii showed 53 percent viability with E. coli $0150: \mathrm{H} 5$ and 62 percent viability with $L$. acidophilus. In none of the above studies cell viability of neither E. coli 0150:H5 (86 percent) nor L. acidophilus (84 percent) got affected by yeast isolates.

\section{Stress tolerance}

Tolerance to temperature by yeast isolates OBS2 and OBS9 was significantly $(\mathrm{p}<0.01)$ different from other isolates at 45 and $40{ }^{\circ} \mathrm{C}$, respectively.. Tolerance observed for OBS2 at $45^{\circ} \mathrm{C}, \mathrm{pH} \mathrm{2,1}$ percent ox-bile and 1 percent organic acid mixture were 97.66, 92.66, 92.33 and 91.33 percent, respectively which were significantly $(\mathrm{p}<0.01)$ different from other yeast isolates (Table 2).

The tolerance ability of yeast isolates to various digestive enzymes such as amylase, pepsin and trypsin was observed and OBS1, OBS2 and S. boulardii showed better tolerance compared to other isolates. However, the percent tolerance observed for OBS2 at $350 \mathrm{IU} \mathrm{ml} \mathrm{m}^{-1}$ amylase, $0.75 \mathrm{mg} \mathrm{ml}^{-1}$ trypsin and $6.0 \mathrm{~g} \mathrm{l}^{-1}$ pepsin was 89.33, 99.33 and 96.66, respectively and significantly $(\mathrm{p}<0.01)$ different from other isolates (Table 3).

Among the 7 yeast isolates, OBS1 and OBS2 tolerate extreme conditions of the gastrointestinal tract like temperature, low $\mathrm{pH}$, organic acids and digestive enzymes, i.e. pepsin and amylase in the stomach and trypsin and bile in the upper intestine.

\section{Pathogenicity of yeast isolates}

The seven yeast isolates did not show any zone of hydrolysis around the colony on specific media indicating that none of the isolates have protease, phospholipase and haemolytic activity (Fig. 3). Further, except OBS1, OBS2 and $S$. boulardii the template DNA was amplified with primers of human pathogenic yeast $C$. albicans and hence were considered as pathogens. The yeast isolates OBS1 and OBS2 were considered as non pathogens and selected for further studies (Fig. 4).

\section{Cell adhesion}

Successful probiotic microorganisms are able to colonize by adhering to the intestinal mucosa at least temporarily. Therefore, we determined the adhesion ability of yeast isolates by following the protocol as mentioned in materials and methods. The mean number of yeast cells was counted and the adherence score for each of the yeast isolate was determined on the basis of size of the inoculum. Among all the yeast isolates, S. boulardii, OBS1 and 

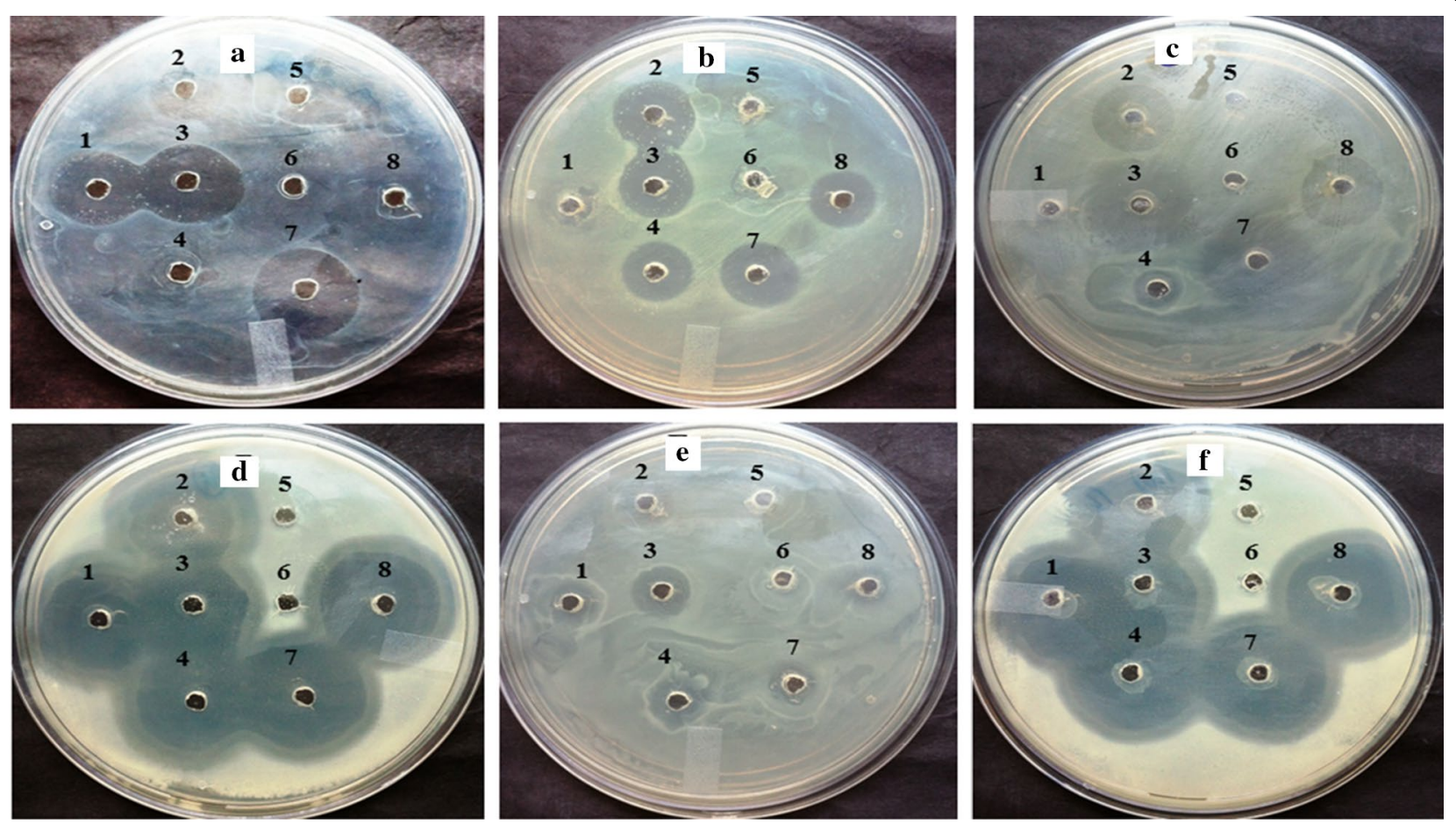

Fig. 2 Antagonistic activity of yeast isolates 1 OBS1, 2 OBS2, 3 OBS3, 4 OBS4, 5 OBS7, 6 OBS9, 7 OBS12 and 8 S. boulardii on human intestinal pathogens a Escherichia coli 0157:H7, b Pseudomonas aeruginosa, c Klebsiella pneumonia, d Staphylococcus aureus, e Salmonella typhi and f Salmonella paratyphi

OBS2 expressed a strong adhesive ability of more than 40 yeast cells adhering to the monolayer.

\section{Molecular characterization}

The yeast isolate OBS2 has $96 \%$ identity and query covers $97 \%$ and E value is zero with Saccharomyces cerevisiae (Fig. 5b), whereas OBS1 has $100 \%$ identity and query covers $68 \%$ and E value is zero with Pichia kudriavzevii (Fig. 5a). The 1370 and 822 base length gene sequences of OBS1 and OBS2 isolates were deposited in the NCBIGenBank data library and acquired accession number KP998094 for S. cerevisiae OBS2 and KP998095 for P. kudriavzevii OBS1. These two yeast isolates were used for assessment of therapeutic properties.

\section{Therapeutic properties}

Out of the two tested yeast samples, sample pertaining to yeast isolate OBS2 resulted in a slight reduction in percentage cell viability of neuroblastoma cell line IMR32
(Fig. 6b) but no reduction in percentage cell viability of breast cancer cell line MCF7 (Fig. 6a) when compared to the control and YPD broth.

The autolysate of yeast isolate OBS1 has shown decreased absorbance i.e. 1.799, 1.685 and 1.542 at $695 \mathrm{~nm}$ with the increased concentration of autolysate i.e. $0.1,0.2$ and $0.3 \mathrm{ml}$ and it was higher than the positive control where it showed 1.642 at $0.3 \mathrm{ml}$ concentration of ascorbic acid. This indicates no antioxidant property. Whereas the autolysate of yeast isolate OBS2 showed increased absorbance i.e. 1.344, 1.454 and 1.528 at $695 \mathrm{~nm}$ with the increased concentration of autolysate i.e. $0.1,0.2$ and $0.3 \mathrm{ml}$ and it was lesser than the positive control indicates it has antioxidant property.

\section{Discussion}

Culturally and morphologically characterized yeast isolates were resistant to majority of the antibacterial drugs, because of their cell wall composition which differs with 


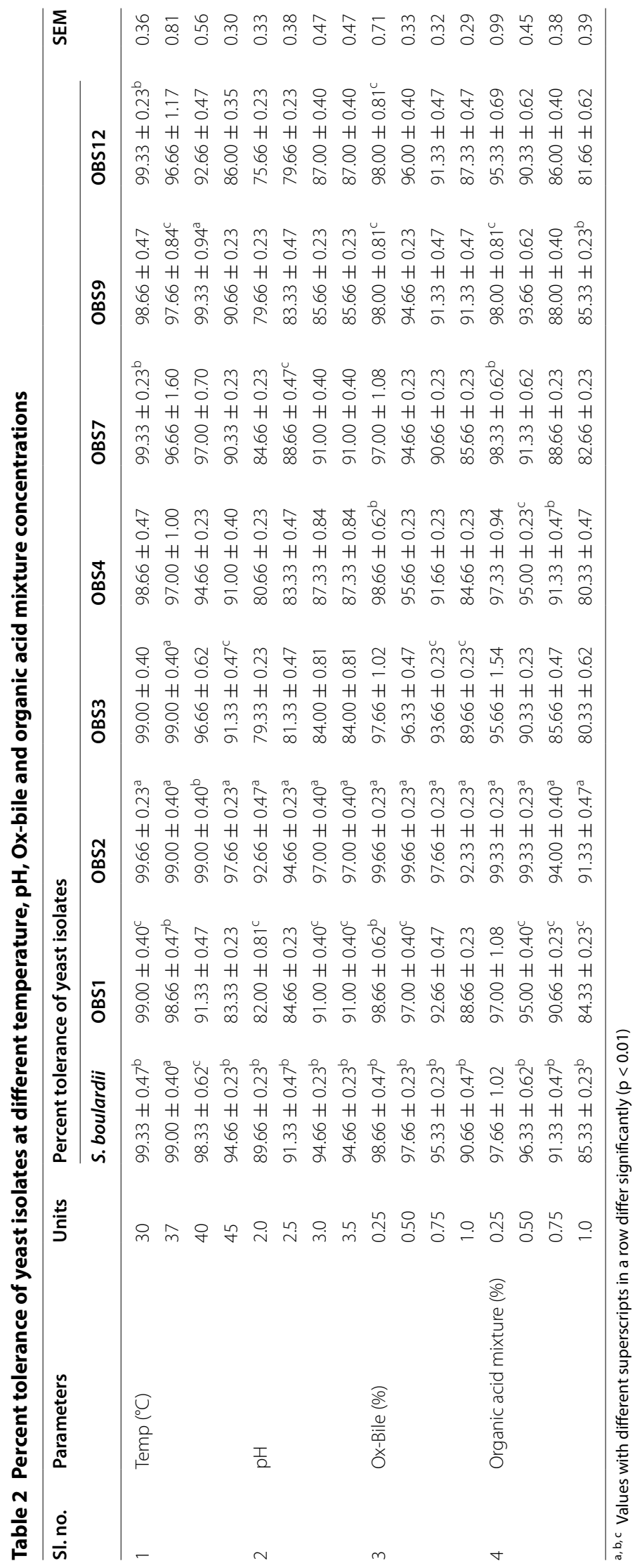




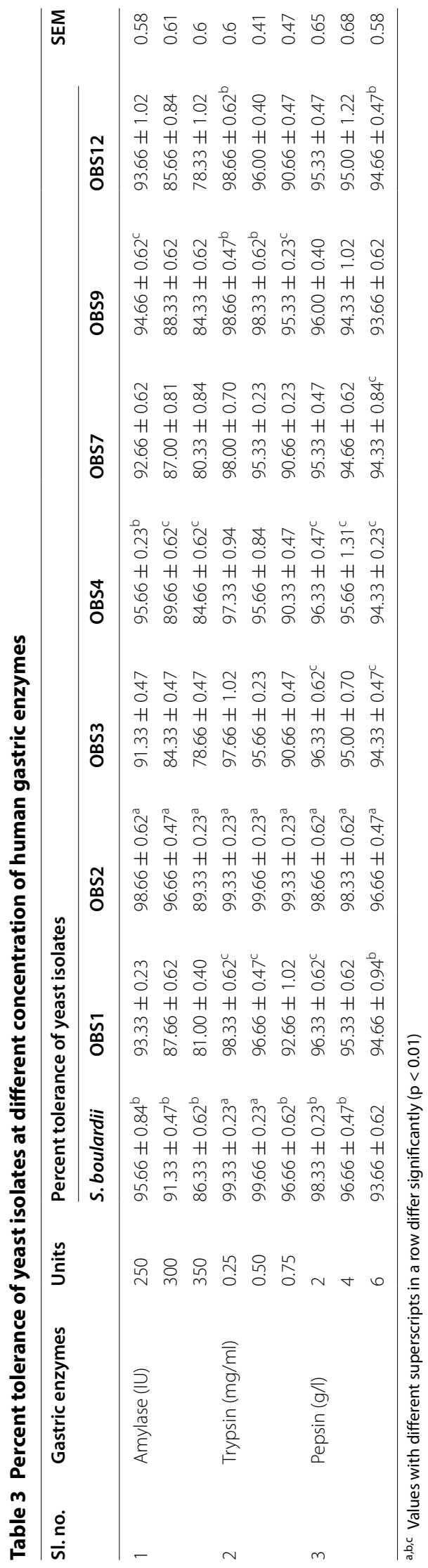



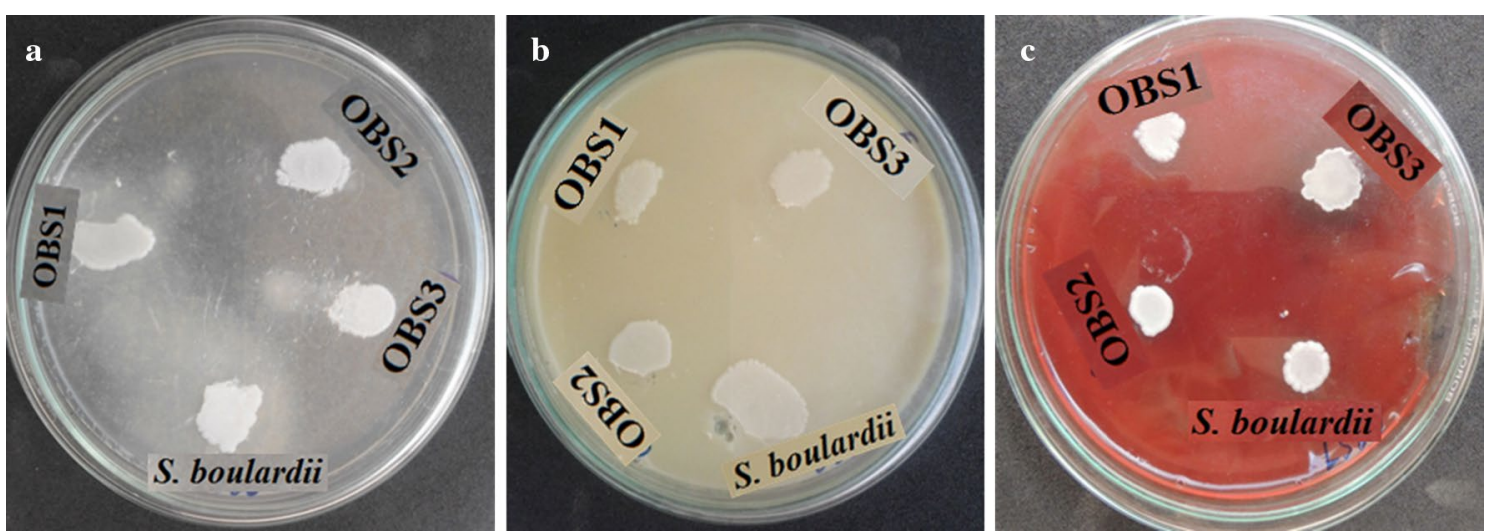

Fig. 3 Pathogenicity of yeast isolates and commercial probiotic S. boulardii: a protease activity, b phospholipase activity and $\mathbf{c}$ haemolytic activity
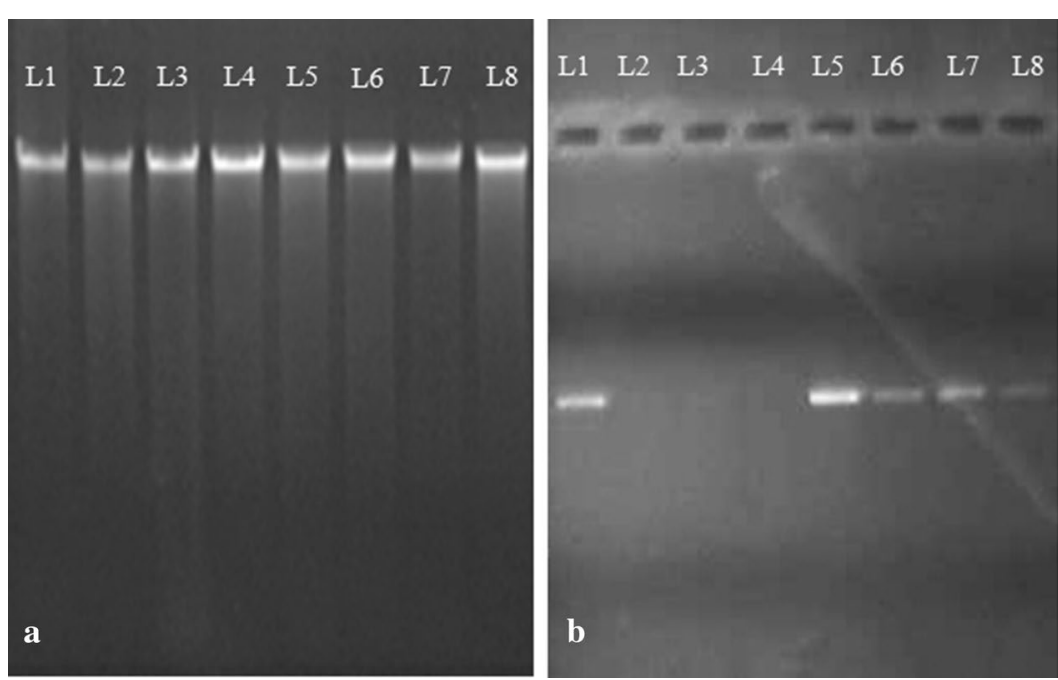

Lane 1: OBS9

Lane 2: S. boulardii

Lane 3: OBS2

Lane 4: OBS1

Lane 5: OBS7

Lane 6: OBS4

Lane 7: OBS3

Lane 8: OBS12

Fig. 4 Pathogenicity of yeast isolates based on gene amplification: $\mathbf{a}$ genomic DNA of the yeast isolates and $\mathbf{b}$ amplicons of yeast isolates

prokaryotes (Czerucka et al. 2007) and most of the isolates were sensitive towards maximum antifungal drugs (Mashad et al. 2012).

Antagonistic activity was observed for $24 \mathrm{~h}$ grown but not $12 \mathrm{~h}$ grown yeast isolates against human intestinal pathogens. When they cultured in a mixed population of intestinal commensals, these isolates showed maximum co-cultural activity (Izadnia et al. 1998; Filho-Lima et al. 2000; Czerucka and Rampal 2002). Antagonistic activity of yeast isolates may be due to the production of antimicrobial metabolites (Hatoum et al. 2012; Rao et al. 2009). The present findings of antagonistic and cocultural properties of yeast isolates are in accordance with Verna and Lucak (2010). The yeast isolates OBS1 and OBS2 were resilient to gastrointestinal parameters is acceptable, when compared with the findings of Kuhle et al. (2005), Bhima et al. (2010), Rajkowska and KunickaStyczynska (2010).

The yeast isolates OBS1 and OBS2 did not show any pathogenic properties and hence confirmed as 


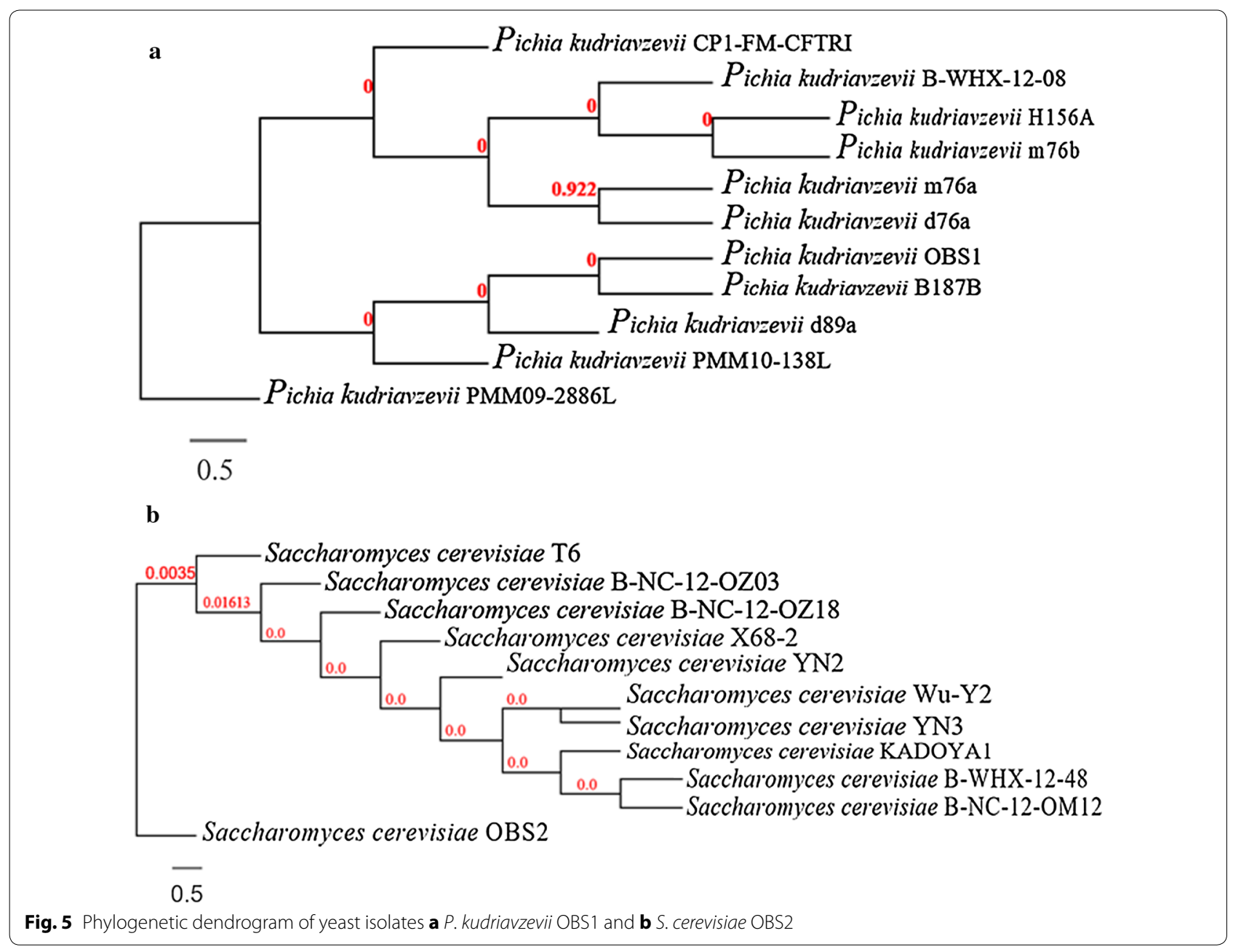

non-pathogens and which is in accordance with the reports of Koehn (1982), Manns et al. (1994), Luo et al. (2001) and Lechuga et al. (2016). Similarly, these isolates have shown strong adhesive property to human intestinal cells (Caco-2). This finding is in accordance with Zivkovic et al. (2015), where the probiotic organism adhesion is more than 40 cells to intestinal cells (Caco-2).

Yeast isolates OBS1 and OBS2 were characterized based on phylogenetic analysis as $P$. kudriavzevii and $S$. cerevisiae. Among the two yeast isolates, OBS2 has a little cytotoxic effect on Neuroblastoma cell line IMR32 which means continuous use of the isolate OBS2 as probiotics may help in curing the incidence of neuroblastoma cancer. On the other hand the same isolate OBS2 has an antioxidant property and by increased cell concentration may improve its probiotic efficiency, which is in agreement with the findings of Naylin et al. (2005) and Oh et al. (2002).

Based on resilience properties to gastrointestinal parameters, antimicrobial agents sensitivity, antagonistic activity, pathogenicity, co-cultural activity, adherence ability to intestinal epithelial cells, 2 yeast isolates were 


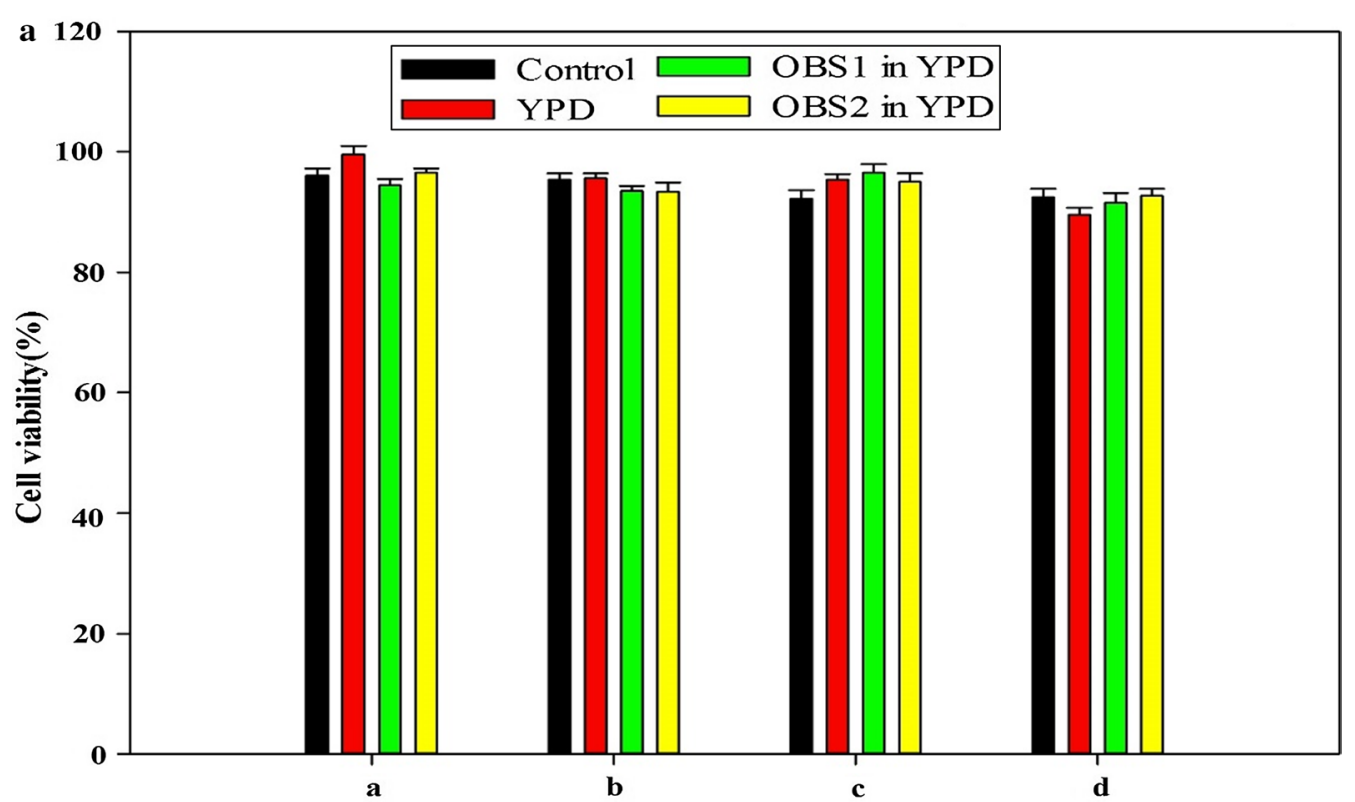

Filtrate tested (a: $1 \mu \mathrm{l}, \mathrm{b}: 10 \mu \mathrm{l}, \mathrm{c}: 20 \mu \mathrm{l}, \mathrm{d}: 40 \mu \mathrm{l})$

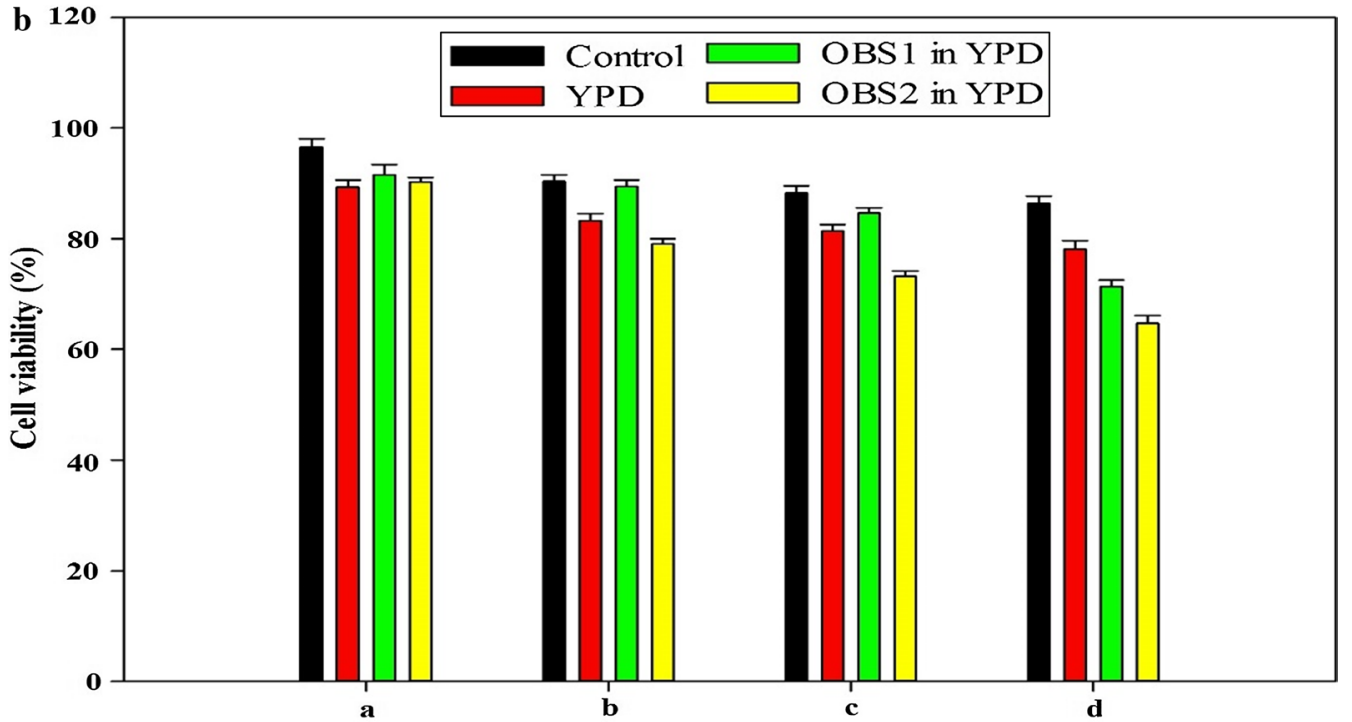

Filtrate tested (a:1 $\mu \mathrm{l}, \mathrm{b}: 10 \mu \mathrm{l}, \mathrm{c}: 20 \mu \mathrm{l}, \mathrm{d:40} \boldsymbol{\mu l})$

Fig. $\mathbf{6}$ Effect of yeast isolates on viability of a breast cancer cell line MCF7 and $\mathbf{b}$ neuroblastoma cell line IMR32

selected and characterized as P. kudriavzevii OBS1 and $S$. cerevisiae OBS2. Of the two isolates $S$. cerevisiae OBS2 has cytotoxic effect on cancer cells and antioxidant activity hence can be used for human probiotic and therapeutic applications.

\section{Abbreviations}

QPS: qualified presumption of safety; YPD: yeast extract peptone dextrose; YNB: yeast nitrogen base; CFU: colony forming units; PBS: phosphate buffered saline; PCR: polymerase chain reaction; NCCS: National Centre for Cell Science; MEM: Eagle's minimal essential medium; ITS: internal transcribed spacer; BLAST: basic local alignment search tool; NCBI: national center for biotechnology information; DMEM: Dulbecco's modified Eagle medium; ANOVA: analysis of variance; DSMZ: Deutsche Sammlung von Mikroorganismen und Zellkulturen $\mathrm{GmbH}$; SRB: sulforhodamine B.

\section{Authors' contributions}

All the experiments were designed and carried out by BS with the help of GSR $B K K$ and $B C$ were involved in procurement of samples from different regions for yeast isolation. KVK carried out the cytotoxicity assays. Co-corresponding author, TAD designed and monitored the cytotoxicity experiments. BB, 
corresponding author designed the concept, monitored all experiments and prepared the manuscript. All authors read and approved the final manuscript.

\section{Author details}

${ }^{1}$ Department of Microbiology, University College of Science, Osmania University, Hyderabad, Telangana 500007, India. ${ }^{2}$ Center for Chemical Biology, CSIR-Indian Institute of Chemical Technology, Tarnaka, Hyderabad, Telangana 500007, India.

\section{Acknowledgements}

Authors are grateful to Science and Engineering Research Board (SERB), Ministry of Science and Technology, Govt. of India for financial support and University Grants Commission, Govt. of India for BSR-RFSMS fellowship to carry out the research work.

\section{Competing interests}

The authors declare that they have no competing interests.

\section{Consent for publication}

This article does not contain any individual person's data.

\section{Declaration}

We declare that this manuscript has not been published elsewhere and is not under consideration by another journal. All authors have approved the final manuscript and agreed with the submission to "AMB Express".

\section{Ethical approval}

This article does not contain any studies concerned with experimentation on human or animals.

\section{Funding}

Science and Engineering Research Board (SERB), Ministry of Science and Technology, Govt. of India (YSS/2015/000173, SB/EMEQ-258/2013).

Received: 21 July 2016 Accepted: 7 December 2016

Published online: 03 January 2017

\section{References}

Abdel Fattah WR, Fadil M, Nigam P, Banat IM (2000) Isolation of thermotolerant ethanologenic yeasts and use of selected strains in industrial scale fermentation in an Egyptian distillery. Biotechnol Bioeng 68(5):531-535

Balasubramanian K, Ragunathan R (2012) Study of antioxidant and anticancer activity of natural sources. J Nat Prod Plant Resour 2(1):192-197

Bhima B, Sudhakara Reddy M, Anjana Devi T, Ramana Reddy Y, Venkateshwar Rao L (2010) Screening and characterization of stress tolerant Saccharomyces cerevisiae isolated from brewery effluents for animal probiotic applications. IIOAB J 1(4):32-39

Blehaut H, Massot J, Elmer GW, Levy RH (1989) Disposition kinetics of Saccharomyces boulardii in man and rat. Biopharm Drug Dispos 10:353-364

Chana Z, Tian S (2005) Interaction of antagonistic yeasts against postharvest pathogens of apple fruit and possible mode of action. Postharvest Biol Tec 36:215-223

Corzo G, Gilliland SE (1999) Bile salt hydrolase activity of three strains of Lactobacillus acidophilus. J Dairy Sci 82:472-480

Czerucka D, Rampal P (2002) Experimental effects of Saccharomyces on diarrhoeal pathogens. Microbes Infect 4:733-739

Czerucka D, Piche T, Rampal P (2007) Review article: yeast as probiotics-Saccharomyces boulardii. Aliment Pharmacol Ther 26:767-778

Filho-Lima JV, Vieira EC, Nicoli JR (2000) Antagonistic effect of Lactobacillus acidophilus. Saccharomyces boulardii and Escherichia coli combinations against experimental infections with Shigella flexneri and Salmonella enteritidis subsp. typhimurium in gnotobiotic mice. J Appl Microbiol 88:365-370

Fooks LJ, Gibson GR (2002) Probiotics as modulators of the gut flora. Brit J Nutr 88(1):39-49

Fridkin SK, Jarvis WR (1996) Epidemiology of nosocomial fungal infections. Clin Microbiol Rev 9(4):499-511
Fujita S, Senda Y, Nakaguchi S, Hashimoto T (2001) Multiplex PCR using internal transcribed spacer 1 and 2 regions for rapid detection and identification of yeast strains. J Clin Microbiol 39(10):3617-3622

Hassan HMM (2011) Antioxidant and immunostimulating activities of yeast (Saccharomyces cerevisiae) autolysates. World Appl Sci J 15(8):1110-1119

Hatoum R, Labrie S, Fliss I (2012) Antimicrobial and probiotic properties of yeasts: from fundamental to novel applications. Front Microbiol 3:1-9

Izadnia F, Wong CT, Kocoshis SA (1998) Brewer's yeast and Saccharomyces boulardii both attenuate Clostridium difficile induced colonic secretion in the rat. Dig Dis Sci 43:2055-2060

Koehn RD (1982) Fungi isolated from sea foam collected at North Padre Island Beaches. Southwest Nat 27:17-21

Kuhle AVA, Skovgaard K, Jespersen L (2005) In vitro screening of probiotic properties of Saccharomyces cerevisiae var boulardii and food borne Saccharomyces cerevisiae strains. Int J Food Microbiol 101(1):29-39

Kumaran A, Karunakaran RJ (2007) In vitro antioxidant activity of methanol extracts of five Phyllanthus species from India. LWT-Food Sci Technol 40(2):344-352

Kumura H, Tanoue Y, Tsukahara M, Tanaka T, Shimazaki K (2004) Screening of dairy yeast strains for probiotic applications. J Dairy Sci 87:4050-4056

Lechuga EGO, Zapata IQ, Nino KA (2016) Detection of extracellular enzymatic activity in microorganisms isolated from waste vegetable oil contaminated soil using plate methodologies. Afr J Biotechnol 15(11):408-416

Luo G, Lakshman Samaranayake P, Yau JYY (2001) Candida Species Exhibit differential in-vitro hemolytic activities. J Clin Microbiol 39(8):2971-2974

Manns JM, Mosser DM, Buckley HR (1994) Production of a hemolytic factor by Candida albicans. Infect Immun 62(11):5154-5156

Marinho SA, Teixeira AB, Santos OS, Cazanova RF (2010) Identification of Candida spp. by phenotypic tests and PCR. Braz J Microbiol 41:286-294

Mashad NE, Mona FF, Saudy N, Dalia AS (2012) Susceptibility tests of oropharyngeal Candida albicans from Egyptian patients to fluconazole determined by three methods. Braz J Microbiol 43(1):266-273

Mc Meekin TA, Brown J, Krist K, Miles D (1997) Quantitative microbiology: a basis for food safety. Emerg Infect Dis 3(4):541-549

Naylin N, Taing OK, Hashinaga F, Toshima Y (2005) Antioxidant activity of sugartolerant yeast Zygosaccharomyces rouxii. Food Biotechnol 19:107-111

Nikolic M, Lopez P, Strahinic I, Suarez A (2012) Characterisation of the exopolysaccharide (EPS)-producing Lactobacillus paraplantarum BGCG11 and its non-EPS producing derivative strains as potential probiotics. Int J Food Microbiol 158(2):155-162

O'Neil CE, Keast DR, Fulgoni VL, Nicklas TA (2012) Food sources of energy and nutrients among adults in the US: NHANES 2003-2006. Nutrients 4(12):2097-2120

Oh Y, Osato MS, Han X, Bennett G, Hong WK (2002) Folk yoghurt kills Helicobacter pylori. J Appl Microbiol 93:1083-1088

Ohashi Y, Kazunari U (2009) Health-beneficial effects of probiotics: its mode of action. Anim Sci J 80(4):361-371

Ortiz ME, Bleckwedel J, Raya RR, Mozzi F (2013) Biotechnological and in situ food production of polyols by lactic acid bacteria. Appl Microbiol Biotechnol 97:4713-4726

Paschos T, Xiros C, Christakopoulos P (2015) Simultaneous saccharification and fermentation by co-cultures of Fusarium oxysporum and Saccharomyces cerevisiae enhances ethanol production from liquefied wheat straw at high solid content. Ind Crop Prod 76:793-802

Pennacchia C, Blaiotta G, Pepe O, Villani F (2008) Isolation of Saccharomyces cerevisiae strains from different food matrices and their preliminary selection for a potential use as probiotics. J Appl Microbiol 105(6):1919-1928

Rajkowska K, Kunicka-Styczynska A (2010) Probiotic properties of yeasts isolated from chicken feces and kefirs. Pol J Microbiol 59(4):257-263

Rao RS, Bhadra B, Shivaji S (2009) Isolation and characterization of ethanol-producing yeasts from fruits and tree barks. Lett Appl Microbiol 47(1):19-24

Reis VR, Bassi APG, Da Silva JCG, Antonini SRC (2013) Characteristics of Saccharomyces cerevisiae yeasts exhibiting rough colonies and pseudohyphal morphology with respect to alcoholic fermentation. Braz J Microbiol 44(4):1121-1131

Sibanda T, Mabinya LV, Mazomba N, Akinpelu DA, Bernard K, Olaniran AO, Okoh Al (2010) Antibiotic producing potentials of three freshwater actinomycetes isolated from the Eastern Cape Province of South Africa. Int J Mol Sci 11:2612-2623

Syal P, Vohra A (2013) Probiotic potential of yeasts isolated from traditional Indian fermented foods. Int J Microbiol Res 5(2):390-398 
Szajewska H, Setty M, Mrukowicz J, Guandalini S (2006) Probiotics in gastrointestinal diseases in children: hard and not-so-hard evidence of efficacy. J Pediatr Gastroenterol Nutr 42(5):454-475

Tikka C, Osuru HP, Atluri N, Chakravarthi P (2013) Isolation and characterization of ethanol tolerant yeast strains. Bioinformation 9(8):421-425

Verna CE, Lucak S (2010) Use of probiotics in gastrointestinal disorders: what to recommend? Ther Adv Gastroenterol 3(5):307-319

Waters DJ, Shen S, Kengeri SS, Chiang EC (2012) Prostatic response to supranutritional selenium supplementation: comparison of the target tissue potency of selenomethionine vs. selenium-yeast on markers of prostatic homeostasis. Nutrients 4(11):1650-1663
Yoo JY, Kim SS (2016) Probiotics and prebiotics: present status and future perspectives on metabolic disorders. Nutrients 8(3):1-20

Zaky AS, Tucker GA, Daw ZY, Du C (2014) Marine yeast isolation and industrial application. FEMS Yeast Res 14(6):813-825

Zanello G, Meurens F, Berri M, Salmon H (2009) Saccharomyces boulardii effects on gastrointestinal diseases. Curr Issues Mol Biol 11(1):47-58

Zivkovic CM, Cadez N, Uroic K, Miljkovic M (2015) Evaluation of probiotic potential of yeasts isolated from traditional cheeses manufactured in Serbia and Croatia. J Intercult Ethnopharmacol 4(1):12-18

\section{Submit your manuscript to a SpringerOpen ${ }^{\circ}$ journal and benefit from:}

- Convenient online submission

- Rigorous peer review

- Immediate publication on acceptance

- Open access: articles freely available online

- High visibility within the field

- Retaining the copyright to your article

Submit your next manuscript at $>$ springeropen.com 